\title{
Cloning and characterization of the nicotianamine synthase gene in Eruca vesicaria subsp sativa
}

\author{
B.L. Huang', C. Cheng', G.Y. Zhang ${ }^{1}$, J.J. Su', Y. Zhi' ${ }^{1}$, S.S. Xu' ${ }^{1}$, D.T. Cai', \\ X.K. Zhang' ${ }^{2}$ and B.Q. Huang' ${ }^{1}$ \\ ${ }^{1}$ Hubei Collaborative Innovation Center for Green Transformation of Bio-Resources, \\ College of Life Science, Hubei University, Wuhan, China \\ ${ }^{2}$ Key Laboratory of Biology and Genetic Improvement of Oil Crops, \\ Ministry of Agriculture, Oil Crops Research Institute of the Chinese Academy of \\ Agricultural Sciences, Wuhan, China \\ Corresponding authors: X.K. Zhang / B.Q. Huang \\ E-mail: zhang.xk@139.com / huangbangquan@163.com
}

Genet. Mol. Res. 14 (4): 18121-18130 (2015)

Received August 4, 2015

Accepted October 16, 2015

Published December 22, 2015

DOI http://dx.doi.org/10.4238/2015.December.22.38

\begin{abstract}
Nicotianamine (NA) is a ubiquitous metabolite in plants that bind heavy metals, is crucial for metal homeostasis, and is also an important metal chelator that facilitates long-distance metal transport and sequestration. NA synthesis is catalyzed by the enzyme nicotianamine synthase (NAS). Eruca vesicaria subsp sativa is highly tolerant to $\mathrm{Ni}, \mathrm{Pb}$, and $\mathrm{Zn}$. In this study, a gene encoding EvNAS was cloned and characterized in E. vesicaria subsp sativa. The full-length EvNAS cDNA sequence contained a 111-bp 5'-untranslated region (UTR), a 155-bp 3'-UTR, and a 966-bp open reading frame encoding 322-amino acid residues. The EvNAS genomic sequence contained no introns, which is similar to previously reported NAS genes. The deduced translation of EvNAS contained a well-conserved NAS domain (1-279 amino acids) and an LIKI-CGEAEG box identical to some Brassica NAS and to the LIRLbox in most plant NAS, which is essential for DNA binding. Phylogenetic analysis indicated that EvNAS was most closely related to Brassica rapa
\end{abstract}


NAS3 within the Cruciferae, followed by Thlaspi NAS1, Camelina NAS3, and Arabidopsis NAS3. A reverse transcription-polymerase chain reaction indicated that EvNAS expression was greatest in the leaves, followed by the flower buds and hypocotyls. EvNAS was moderately expressed in the roots.

Key words: Eruca vesicaria subsp sativa; Nicotianamine synthase; Gene cloning

\section{INTRODUCTION}

Nicotianamine (NA) is a key ligand that is involved in metal transport and homeostasis in plants (Inoue et al., 2003; Douchkov et al., 2005). NA is a ubiquitous metabolite in plants that is able to bind heavy metals both in vitro and in vivo, and that is crucial to the homeostasis of essential metals such as iron, zinc, manganese, nickel, and copper (Takahashi et al., 2003; Pianelli et al., 2005). NA is also an important metal chelator that facilitates long-distance metal transport and sequestration (Ling et al., 1996, 1999; Mari et al., 2006; Dreyfus et al., 2009). The synthesis of NA from three molecules of S-adenosyl-L-methionine is catalyzed by the enzyme nicotianamine synthase (NAS; Kawai et al., 1988; Shojima et al., 1989). Vacchina et al. (2003) demonstrated that in yeast cells expressing TcNAS1 cDNA from Thlaspi caerulescens (now Noccaea caerulescens), NA bound nickel in vivo, resulting in a reduction in nickel cytotoxicity. Pianelli et al. (2005) reported enhanced nickel resistance both at the cellular and whole-plant level in Arabidopsis thaliana transgenic lines that had over-accumulated NA. They also observed a direct correlation between the amounts of NA accumulated in planta and the nickel resistance phenotype. Moreover, these plants can accumulate up to $0.25 \%$ dry weight of their aerial parts of nickel on nickel-contaminated soil without any symptom of toxicity. Pianelli et al. (2005) also demonstrated that improved nickel tolerance can be achieved by increasing resistance at the cellular level by NA accumulation, and that such a strategy can be envisaged for phytoremediation purposes.

The results of our previous study indicated that Eruca is highly tolerant to $\mathrm{Ni}, \mathrm{Zn}$, and $\mathrm{Pb}$ (Zhi et al., 2015). Here, we report the cloning and characterization of full-length cDNA and genomic DNA sequences of the EvNAS gene from Eruca vesicaria subsp sativa. The expression profiles of EvNAS in different tissues were also examined.

\section{MATERIAL AND METHODS}

\section{Plants and tissue collection}

E. vesicaria subsp sativa 'hubu-14' (E. sativa 'hubu-14'), which is highly tolerant to $\mathrm{Ni}, \mathrm{Zn}$, and $\mathrm{Pb}$ (Zhi et al., 2015), was used for cloning the NAS gene. Seeds were germinated on filter paper immersed in liquid Murashige and Skoog medium without sugar or organic components. Seven days after seed inoculation, the roots and hypocotyls were harvested separately and frozen immediately in liquid nitrogen and stored at $-80^{\circ} \mathrm{C}$. Young leaves and flower buds were collected from the field and also frozen immediately in liquid nitrogen and stored at $-80^{\circ} \mathrm{C}$.

\section{Cloning of full-length cDNA and EvNAS genomic DNA}

Total RNA was isolated using TRIzol ${ }^{\circledR}$ Total RNA Extraction Reagent (Takara) according to 
the manufacturer protocol. First-strand cDNA was synthesized with $1 \mu \mathrm{g}$ total RNA and $1 \mu \mathrm{L}$ ReverTra Ace $^{\circledR}$ (100 U, Toyobo) following the manufacturer instructions. NAS sequences from different higher plants (Arabidopsis halleri, JQ619642.1; Arabidopsis thaliana, AB181237.1; Brassica rapa, XM_009144131; Camelina sativa, XM_010425211.1; Citrus sinensis, XM_006472133.1; Cucumis melo, XP_008453616.1; Lotus japonicas, BAH22562.1; Malus baccata, ABD64879.1; Medicago truncatula, XP_003591220.1; Nicotiana tomentosiformis, XP_009619408.1; Nicotiana sylvestris, XP_009804361.1; Noccaea caerulescens, JF714218.1; Solanum lycopersicum, XP_004230307.1; and Tarenaya hassleriana, XM_010545902) were aligned by ClustalW2 (http://www.ebi.ac.uk/ Tools/msa/clustalw2/), and some highly conserved regions were retrieved. A pair of degenerated primers (NAS core-FP and NAS core-RP; Table 1) was designed according to these highly conserved regions to amplify a 284-bp fragment of the NAS coding region using leaf and root cDNAs as templates. Two gene-specific primers (5'-RACE-NAS and 3'-RACE-NAS; Table 1) were designed for 5'-rapid amplification of cDNA ends (RACE) and 3'-RACE to clone full-length EvNAS cDNA using a SMARTer ${ }^{T M}$ RACE Amplification Kit (Clontech). Primers (NAS-FP and NAS-RP; Table 1) based on sequences of the 5'-RACE and 3'-RACE fragments were used to amplify the full-length EVNAS from cDNA and genomic DNA. All of the polymerase chain reaction (PCR) products were cloned into a pMD-18T vector (Takara) and sequenced. At least five independent clones were sequenced for each PCR product to ensure sequencing accuracy.

Table 1. Primers and sequences used in the study.

\begin{tabular}{lllr}
\hline PCR system & Primer name & Primer sequence (5'-3') & Amplicon size/bp \\
\hline Core fragment PCR & NAS core-FP & TCACGTCCATCGTTCTTGCTTCA & 284 \\
& NAS core-RP & AGCACCAGGAGCCATGTGTTTCTG & 674 \\
5'-RACE & 5'-RACE-NAS & GGGCAAGCAGTGGTATCAACGCAGAGT & 741 \\
3'-RACE & 3'-RACE-NAS & GTACTCTGCGTTGATACCACTGCTTGC & 1143 \\
cDNA full-length PCR & NAS-FP & GGGGACCCGAGAAACACAATAT & 1143 \\
& NAS-RP & ACATTACACCAACATACAACACAAAGAC & \\
Genomic DNA PCR & NAS-FP & GGGGACCCGAGAAACACAATAT & 277 \\
qRT-PCR & NAS-RP & ACATTACACCAACATACAACACAAAGAC & \\
& NAS qRT-FP & CGTCGTGTTTCTAGCAGCTCTCGTTGG & \\
& NAS qRT-RP & AGCACGATGGCCCACCTAAAATACTCTC & \\
& actin qRT-FP & CGCCGCTTAACCCTAAGGCTAACAG & \\
\hline
\end{tabular}

PCR, polymerase chain reaction; RACE, rapid amplification of CDNA ends; qRT-PCR, quantitative reverse transcriptionpolymerase chain reaction.

\section{Sequence analysis}

ORF Finder (http://www.ncbi.nlm.nih.gov/gorf/gorf.html) was used to perform open reading frame (ORF) analysis and deduce the amino acid sequence. The genomic organization of the EvNAS genomic DNA was determined using Splign (http://www.ncbi.nlm.nih.gov/sutils/ splign/splign.cgi). The protein theoretical isoelectric point $(\mathrm{pl})$ and molecular weight $(\mathrm{Mw})$ were computed using the Compute pl/Mw tool (http://web.expasy.org/compute_pi/). Nucleotides, as well as the derived amino acid sequences of EVNAS, were BLASTP-searched with reported NAS gene sequences of different species using the National Center for Biotechnology Information (NCBI) server (http://www.ncbi.nlm.nih.gov/BLAST). A motif scan of the deduced EvNAS protein was conducted using the Pfam database (http://pfam.sanger.ac.uk/), the secondary structure was predicted by SOPMA (http://npsa-pbil.ibcp.fr/), and a phylogenetic tree was constructed using DNAMAN (Lynnon Biosoft). 


\section{Expression analysis by real-time quantitative reverse transcription-PCR (qRT-PCR)}

To investigate the EvNAS mRNA expression profile, total RNA from leaves, roots, hypocotyls, and flower buds were extracted for real-time qRT-PCR. The constitutively expressed actin gene was used as an internal control to normalize the transcript levels of the target gene. The primers described in Table 1 (NAS qRT-FP and NAS qRT-RP) were used to detect EvNAS expression levels. The qRT-PCRs were performed in triplicate according to the manufacturer (Bioer) instructions using the comparative $\mathrm{Ct}$ method, and the results are presented as means and standard deviations.

\section{Statistical analysis}

Statistical analysis was conducted using the SPSS software (version 18.0, SPSS Inc., Chicago, IL, USA). Spearman's rank correlation was used to assess relationships between EVNAS expression levels in different tissues of $E$. vesicaria subsp sativa, and analysis of variance was conducted to identify gene transcripts that exhibited significant $(P<0.05)$ changes in relative abundance in the different tissues.

\section{RESULTS}

\section{Cloning and sequence analysis of EvNAS}

By using degenerated primers based on highly conserved sequences of NAS from different species, a 284-bp conserved fragment was amplified from E. vesicaria subsp sativa root, hypocotyl, leaf, and flower bud mRNA. By aligning and splicing the sequences of the conserved region, a 1235-bp full-length EVNAS cDNA was predicted to contain a 111-bp 5'-untranslated region (UTR), a 966-bp coding region, and a 155-bp 3'-UTR (Figure 1). A 1143-bp sequence was amplified from cDNA and genomic DNA using primers based on 3'- and 5'-RACE sequences. ORF Finder indicated that the EvNAS cDNA sequence contained a 966-bp ORF.

\section{Analysis of deduced amino acid sequence}

The pl of the EvNAS protein was 5.70 and the Mw was $35954.75 \mathrm{Da}$. A comparison of amino acid sequence homologies and biochemical properties of NAS from different plant species is provided in Table 2 .

The BLASTP analysis revealed that the deduced amino acid sequence of EvNAS shared the highest identity (94.1\%) with B. rapa NAS3 (GenBank accession No. XM_009149927.1), followed by T. caerulescens NAS1 (AJ300446.1, 90.4\% identity), C. sativa NAS3 (XM_010459903.1, 89.4\% identity), A. halleri NAS2 (JQ619642.1, 87.3\% identity), and A. halleri NAS3 (AJ580399.1, 87.0\% identity). The putative secondary structure of the deduced amino acid sequence is presented in Figure 2.

Multiple alignments of the EvNAS protein with NAS from other species are presented in Figure 3. The motif scan in Pfam confirmed that the EvNAS protein contains an intact NAS domain (1-279 amino acids) and a LIKI-CGEAEG box identical to some Brassica NAS and similar to the LIRL-box of most plant NAS. 


\begin{tabular}{|c|c|}
\hline 1 & ACATGCCGA COCGAGAAACACAATA TCTTGAAGAAAAAAAAATAA CAAGAACATATAAAT \\
\hline 61 & TTGICCTATTTTA TTTTTCCTTTCAAAACAAAATACCCTCA TAGTGICGAdATGGATTCC \\
\hline & M D \\
\hline 21 & CCAGA CGAGCAACTCGTCAAAACAA TTTGOCATCTCTA CGA GAAGATCTCAAAGCTCAAG \\
\hline & 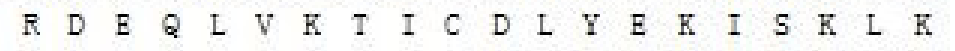 \\
\hline 1 & AGTCTAAAA CCATCOCAAGATGICAACATTCTCTTCAGCCA GCTCGICTCCACA TCCATC \\
\hline & 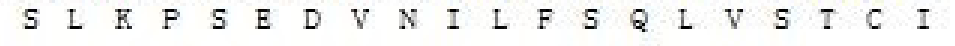 \\
\hline 1 & CCACCCAACCCTAACATCGACGICA CCAA GATGTGIGA CACAGTCCAAGACACT CGICAA \\
\hline & 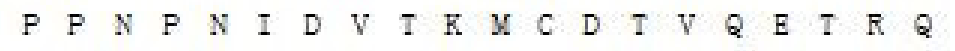 \\
\hline & AAACTCATCAAGA TCTGTCGTGAAGCTCAAGGICACCTACA ACATCATTTCTCTTCCA TC \\
\hline 1 & 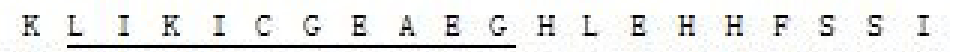 \\
\hline 1 & TTCACGTCTTTTGAACA CAAC CCACTTAA CCATTTAAA CATTTTC CCTTATTACAATAAC \\
\hline 1 & 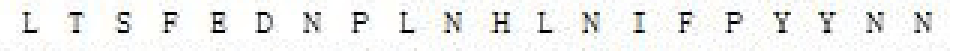 \\
\hline & TATATAAAA CTCGOCAAGCTCGAGTACGA TCTOCTCACACAAAACCTAAACCGICTTGTC \\
\hline & 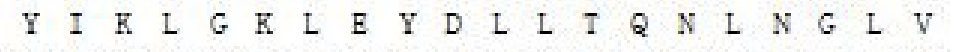 \\
\hline & CCAAA GACA GTTGCTTTCGTT GCATCOCGTCCTCTTCC TTTAACTTCCA TCGTTCTTGCT \\
\hline & 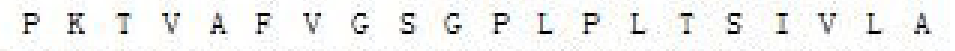 \\
\hline 1 & TCGTCTCATCTCAAAGA CACA GICTTTCA CAACTTTCA CATCCACCATTCACCGAACTCA \\
\hline & 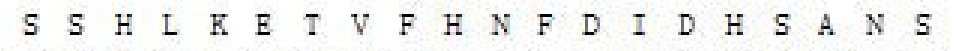 \\
\hline & CTCCCTTCTCTTCTCGITTCTTCTGATCCACACATCTCTCAACCCATGTTCTTCCACACC \\
\hline & L A S L L V S S D P D I S Q R Y F F H I \\
\hline 1 & GTTCA TATAATCGAAGTGACAGACA CCTTAAAGACCTTCGA CGICGIGITTCTAGCAGCT \\
\hline & 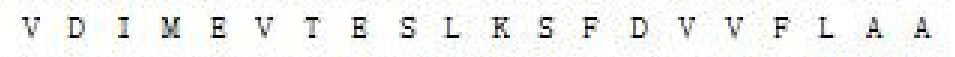 \\
\hline & CTTGTTCGGATCAACAAGCAGGATAAAGT TAAAGTCATCCA GCATCTTCAGAAACACATG \\
\hline & 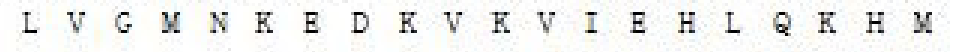 \\
\hline 1 & GCTCCTCGICCTGTACTCATGCTCA CGAGTCCTCATCGTCCTAGAGCGITTCTTTATCCG \\
\hline 51 & 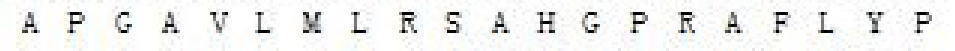 \\
\hline & ATCGTTCAGCOCTGICA TCTTCAAGGGTTCGAGGTTTGGCTATCTATCATCOGACGGAT \\
\hline & 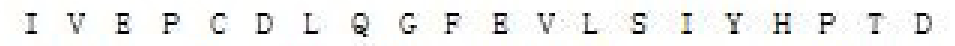 \\
\hline 1 & CATGTTATCAACTOCGIGGTGATCTCGAAAAACCTCOCTGTTGICTCAAATCAGAGTATT \\
\hline 1 & 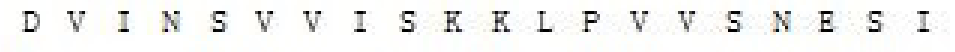 \\
\hline 1 & TTACGTCGGOCATCGTGCTTGCTTA TCOCTTGTACCTGTTCCAAGATCCATCCTATAATG \\
\hline & 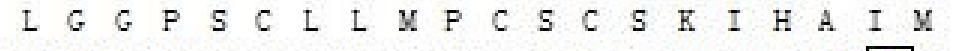 \\
\hline 21 & AACAAGAAGAAGAACATCATGATCGAGGAGTTCGCAGCCAGGCAAGAACAGTTT- \\
\hline 1 & 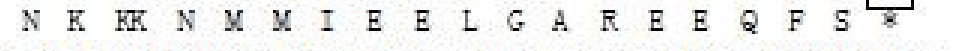 \\
\hline 81 & GATCA CGTTATTTCTTAATTCACTA TCTTAATAATGATTTGCGTGTCTTTGTGTTGT. \\
\hline & TTCGTGTAA TGTTTCTA TCCA TTGTTCTCTCGTTTGTTATAACTTATAAAAGTG \\
\hline & 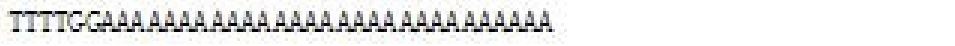 \\
\hline
\end{tabular}

Figure 1. Full-length NAS cDNA sequence of Eruca vesicaria subsp sativa and the deduced amino acid sequence. The initiator codon and stop codon are boxed, and sequences before the initiator codon and after the stop codon are the 5'-untranslated region (UTR) and the 3'-UTR, respectively. The LIKI-CGEAEG box is underlined. 
Table 2. Homologies of amino acid sequences and biochemical properties of nicotianamine synthase (NAS) in different plant species.

\begin{tabular}{|c|c|c|c|c|c|c|}
\hline NAS from plants & Accession No. & No. of amino acid & Similarity (\%) & Identity (\%) & Molecular weight & Isoelectric point \\
\hline Eruca vesicaria subsp sativa NAS & & 322 & - & - & $35,954.75$ & 5.7 \\
\hline Brassica rapa NAS3 & XM_009149927.1 & 323 & 96.0 & 94.1 & $36,076.79$ & 5.51 \\
\hline Thlaspi caerulescens NAS1 & AJ300446.1 & 321 & 93.8 & 90.4 & $35,896.92$ & 5.7 \\
\hline Camelina sativa NAS3 & XM_010459903.1 & 322 & 92.5 & 89.4 & $35,857.66$ & 5.52 \\
\hline Arabidopsis halleri NAS2 & JQ619642.1 & 320 & 90.7 & 87.3 & $35,683.44$ & 5.85 \\
\hline Arabidopsis halleri NAS3 & AJ580399.1 & 320 & 90.1 & 87.0 & $35,749.47$ & 5.69 \\
\hline Arabidopsis thaliana NAS4 & AB181237.1 & 324 & 84.3 & 74.4 & $36,349.59$ & 5.08 \\
\hline Brassica rapa NAS1 & XM_009144131.1 & 323 & 83.3 & 74.4 & $36,207.38$ & 5.23 \\
\hline Noccaea caerulescens NAS4 & JF714218.1 & 322 & 82.7 & 74.4 & $36,201.37$ & 5.12 \\
\hline Solanum lycopersicum NAS & NP_001296307 & 317 & 74.4 & 59.6 & $35,300.68$ & 5.72 \\
\hline Arabidopsis thaliana NAS1 & AED90808.1 & 320 & 73.2 & 64.0 & $35,544.93$ & 6.15 \\
\hline Camelina sativa NAS1 & XM_010425211.1 & 321 & 72.4 & 63.2 & $35,670.86$ & 5.95 \\
\hline Arabidopsis thaliana NAS2 & NP_200419.1 & 320 & 72.3 & 63.1 & $35,676.79$ & 6.05 \\
\hline Arabidopsis thaliana NAS3 & NM_-100794 & 320 & 72.0 & 64.0 & $35,574.78$ & 6.04 \\
\hline Malus baccata NAS2 & ABD-64879.1 & 325 & 72.0 & 58.2 & $36,090.44$ & 5.33 \\
\hline Brassica rapa NAS2 & XM_009128720.1 & 320 & 71.7 & 63.4 & $35,745.13$ & 5.62 \\
\hline Camelina sativa NAS2 & XP_010450614.1 & 320 & 71.7 & 63.4 & $35,555.58$ & 5.85 \\
\hline Zea mays NAS2 & NP_001104862.1 & 327 & 62.8 & 47.7 & $35,621.68$ & 6.11 \\
\hline Brassica rapa NAS4 & XP_009122978.1 & 278 & 61.1 & 50.3 & $31,456.48$ & 6.63 \\
\hline Hordeum vulgare NAS6 & BAĀ74586.1 & 328 & 61.0 & 46.7 & $35,349.55$ & 5.18 \\
\hline Oryza sativa japonica NAS3 & BAF22621.1 & 343 & 60.7 & 44.7 & $36,981.03$ & 5.53 \\
\hline Hordeum vulgare NAS4 & BAA74583.1 & 329 & 60.4 & 46.2 & $35,395.36$ & 5.04 \\
\hline Hordeum vulgare NAS7 & BAA74587.1 & 329 & 59.9 & 45.7 & $35,243.39$ & 5.1 \\
\hline Hordeum vulgare NAS2 & AAD32651.1 & 340 & 59.8 & 42.9 & $36,310.46$ & 5.18 \\
\hline Hordeum vulgare NAS3 & BAA74581.1 & 335 & 59.8 & 45.6 & $3,612.35$ & 5.53 \\
\hline Hordeum vulgare NAS1 & BAA74580.1 & 328 & 59.7 & 44.2 & $35,143.8$ & 5.3 \\
\hline Oryza sativa japonica NAS2 & BAF11808.1 & 326 & 59.5 & 45.0 & $34,483.94$ & 5.56 \\
\hline Oryza sativa japonica NAS1 & BAF11809.1 & 332 & 58.4 & 43.4 & $34,930.33$ & 5.08 \\
\hline Zea mays NAS1 & NP_001105504.1 & 364 & 53.4 & 39.6 & $39,030.33$ & 5.42 \\
\hline Zea mays NAS3 & NP_001151345.1 & 601 & 33.8 & 25.5 & $65,822.96$ & 6.08 \\
\hline
\end{tabular}

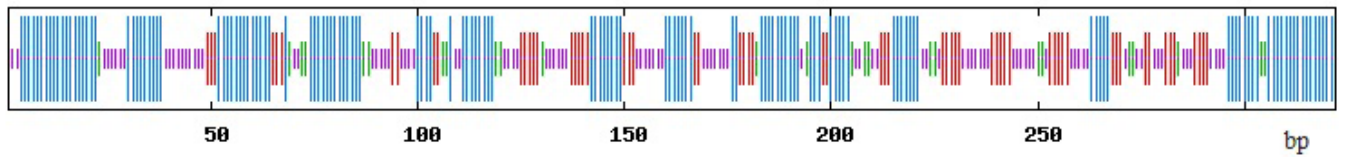

Figure 2. Secondary structure of EvNAS predicted by SOPMA. The longest, second longest, third longest, and shortest lines represent alpha helixes, extended strands, beta turns, and random coils, respectively.

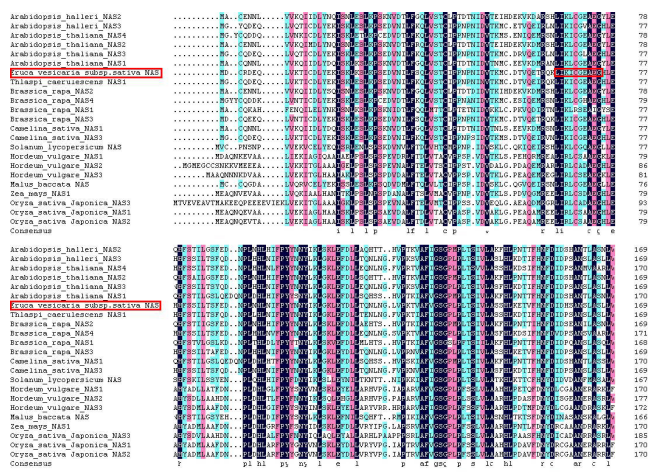

Figure 3. Multiple alignments of the $\mathrm{N}$-terminus of nicotianamine synthase (NAS) proteins from various plant species. The Eruca NAS and LIKI-CGEAEG are presented in the boxes. 


\section{Sequence alignments and phylogenetic analysis}

As shown in the phylogenetic tree (Figure 4), the NAS proteins were clustered into two main groups: NAS from monocots (Group M) and NAS from dicots (Group D). The monocot group included NAS from Elaeis, Hordeum, Oryza, and Zea mays. In the dicot group, EvNAS and the other Cruciferae NAS were clustered with two Tarenaya NAS and formed a separate subgroup (subgroup C). Within the Cruciferae, EvNAS was most closely related to B. rapa NAS3, followed by Thlaspi NAS1, Camelina NAS3, and Arabidopsis NAS3.

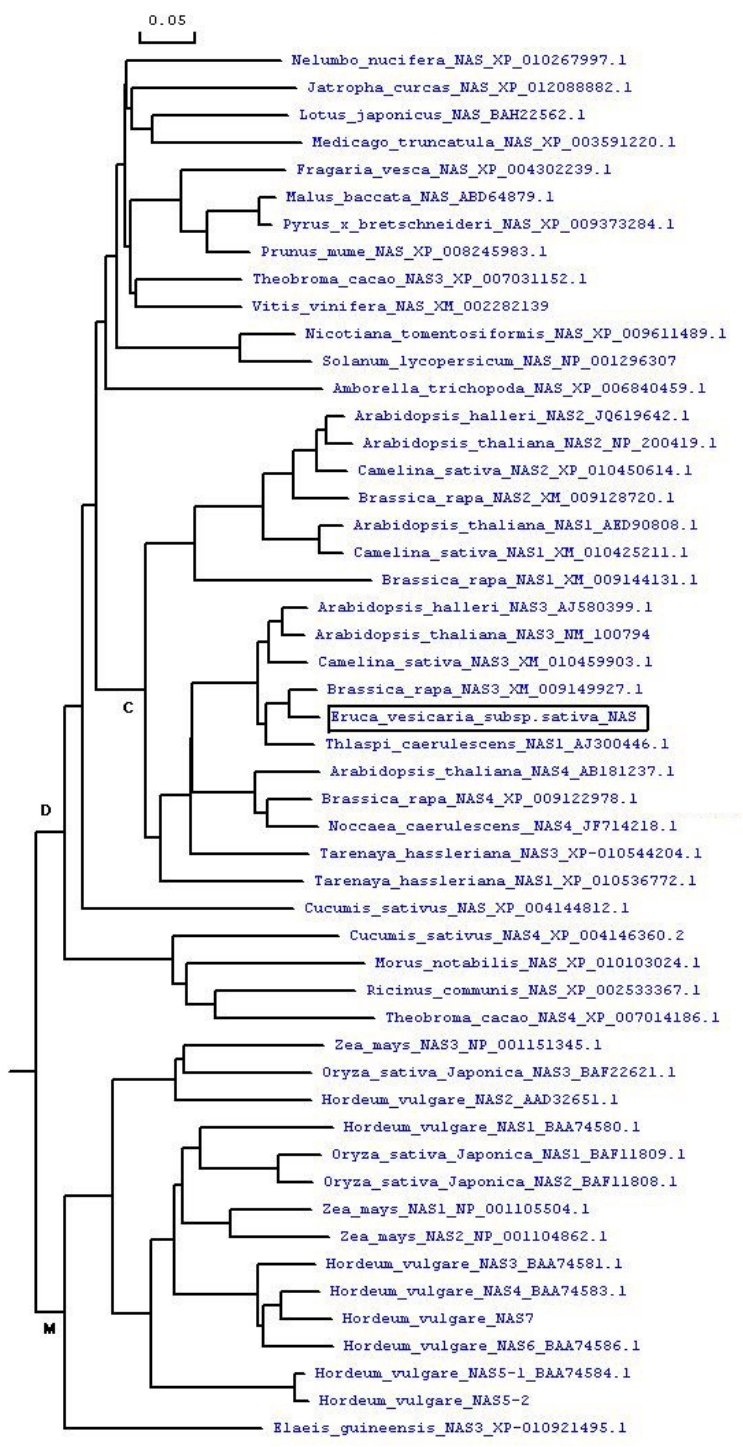

Figure 4. Phylogenetic relationships between EvNAS and nicotianamine synthase (NAS) proteins from different plant species. The Eruca NAS is boxed. 


\section{Tissue-specific expression of EvNAS}

In this study we found that EvNAS was expressed at different levels in different tissues of $E$. vesicaria subsp sativa and was highest in the leaf, followed by the flower bud and hypocotyl. The EVNAS expression level in the roots was moderate; indeed, the EVNAS expression level in the leaves was almost three times higher than that in the roots (Figure 5).

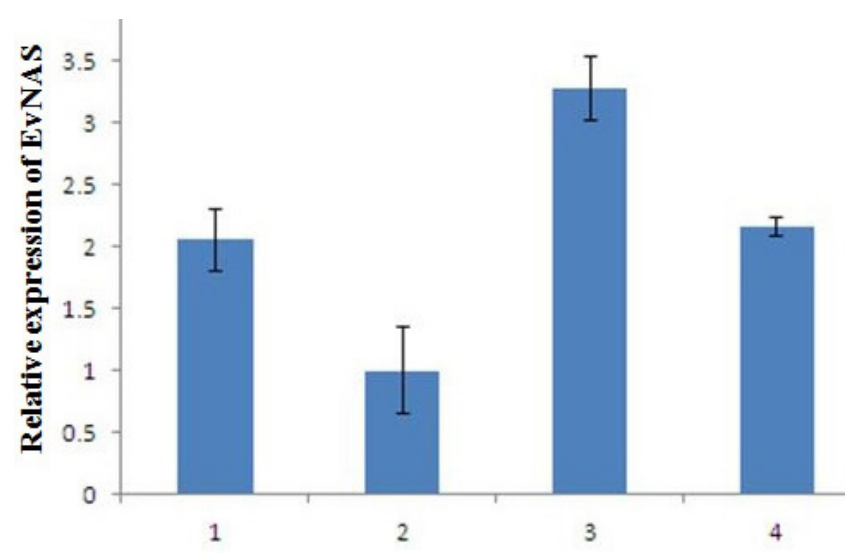

Figure 5. Relative mRNA expression levels (means \pm standard deviations) of Eruca vesicaria subsp sativa nicotianamine synthase. The expression of EvNAS in hypocotyl is normalized as 1.0 and that in root, leaf and flower bud is presented as relative to that in hypocotyl. Data are reported as means \pm SD. 1 , root; 2 , hypocotyl; 3 , leaf; 4 , flower bud.

\section{DISCUSSION}

In this study, we cloned full-length NAS cDNA and genomic DNA sequences from $E$. vesicaria subsp sativa. The EvNAS genomic sequence contained no introns, which is similar to that previously reported in other species (Ling et al., 1999; Higuchi et al., 2001; Mizuno et al., 2003; Zhou et al., 2013). The deduced translation of EVNAS contained a well-conserved NAS domain (1279 amino acids) and a LIKI-CGEAEG box identical to some Brassica NAS and similar to the LIRLbox of most plant NAS, which is essential for DNA binding (Yang et al., 2015). In the phylogenetic tree (Figure 4) EvNAS and the other Cruciferae NAS were clustered with two Tarenaya NAS and formed a separate subgroup (subgroup C). This was to be expected, because Tarenaya and the Cruciferae species belong to the Capparales. Within the Cruciferae, EvNAS was most closely related to B. rapa NAS3, followed by Thlaspi NAS1, Camelina NAS3, and Arabidopsis NAS3. Higuchi et al. (1999) reported that the expression of NAS in barley is induced by Fe deficiency and is root specific, while OsNAS1 expression in rice is induced by Fe deficiency in both the roots and shoots (Ito et al., 2009). In maize, a RT-PCR revealed that all $10 \mathrm{ZmNAS}$ genes were only observed in root tissue, except for ZmNAS6 (Zhou et al., 2013). In tomato, NAS is expressed in the leaves and roots regardless of Fe status (Ling et al., 1999). In A. thaliana, AtNAS1 and AtNAS2 have been detected in both shoots and roots, while AtNAS3 was present only in the shoots (Suzuki et al., 1999). In A. halleri, NAS1 and NAS4 are expressed in both the roots and the leaves, while NAS2 is predominantly expressed in the roots and NAS3 almost exclusively in the leaves (Deinlein et al., 2012). In Malus xiaojinensis, MxNas1 expression is enriched in the roots and leaves (Han 
et al., 2013), while MxNAS2 expression is more enriched in the leaf, root, and phloem than in the xylem (Yang et al., 2015). Here in this study we found that the expression of EvNAS highest in the leaf, followed by the flower bud and hypocotyl. The EVNAS expression level in the roots was moderate.

\section{Conflicts of interest}

The authors declare no conflict of interest.

\section{ACKNOWLEDGMENTS}

Research supported by the Science and Technology Department of Hubei Province, the Huangshi Science and Technology Bureau, the Key Laboratory of Biology and Genetic Improvement of Oil Crops, Ministry of Agriculture, China, the National Natural Science Foundation of China (\#30771382, \#30671334, \#30971807, and \#31201238), the European Committee 7th Framework Program (\#ICON, 211400), and the Swedish Research Links Project.

\section{REFERENCES}

Deinlein U, Weber M, Schmidt H, Rensch S, et al. (2012). Elevated nicotianamine levels in Arabidopsis halleri roots play a key role in zinc hyperaccumulation. Plant Cell 24: 708-723.

Douchkov D, Gryczka C, Stephan UW, Hell R, et al. (2005). Ectopic expression of nicotianamine synthase genes results in improved iron accumulation and increased nickel tolerance in transgenic tobacco. Plant Cell Environ. 28: 365-374.

Dreyfus C, Lemaire D, Mari S, Pignol D, et al. (2009). Crystallographic snapshots of iterative substrate translocations during nicotianamine synthesis in Archaea. Proc. Natl. Acad. Sci. U. S. A. 106: 16180-16184.

Han DG, Yang GH, Xu KD, Shao Q, et al. (2013). Over-expression of a Malus xiaojinensis Nas1 gene influences flower development and tolerance to iron stress in transgenic tobacco. Plant Mol. Biol. Rep. 31: 802-809.

Higuchi K, Suzuki K, Nakanishi H, Yamaguchi H, et al. (1999). Cloning of nicotianamine synthase genes, novel genes involved in the biosynthesis of phytosiderophores. Plant Physiol. 119: 471-479.

Higuchi K, Watanabe S, Takahashi M, Kawasaki S, et al. (2001). Nicotianamine synthase gene expression differs in barley and rice under Fe-deficient conditions. Plant J. 25: 159-167.

Inoue H, Higuchi K, Takahashi M, Nakanishi H, et al. (2003). Three rice nicotianamine synthase genes, OsNAS1, OsNAS2, and OsNAS3 are expressed in cells involved in long-distance transport of iron and differentially regulated by iron. Plant J. 36: 366-381.

Ito S, Inoue H, Kobayashi T, Yoshiba M, et al. (2009). Comparison of the functions of the barley nicotianamine synthase gene HvNAS1 and rice nicotianamine synthase gene OsNAS1 promoters in response to iron deficiency in transgenic tobacco. Soil Sci. Plant Nutr. 55: 277-282.

Kawai S, Itoh K, Takagi SI, Iwashita T, et al. (1988). Studies on phytosiderophores: biosynthesis of mugineic acid and 2'-deoxymugineic acid in Hordeum vulgare L. var. Minorimugi. Tetrahedron Lett. 29: 1053-1056.

Ling HQ, Pich A, Scholz G and Ganal MW (1996). Genetic analysis of two tomato mutants affected in the regulation of iron metabolism. Mol. Gen. Genet. 252: 87-92.

Ling HQ, Koch G, Baumlein H and Ganal MW (1999). Map-based cloning of chloronerva, a gene involved in iron uptake of higher plants encoding nicotianamine synthase. Proc. Natl. Acad. Sci. USA 96: 7098-7103.

Mari S, Gendre D, Pianelli K, Ouerdane L, et al. (2006). Root-to-shoot long-distance circulation of nicotianamine and nicotianamine-nickel chelates in the metal hyperaccumulator Thlaspi caerulescens. J. Exp. Bot. 57: 4111-4122.

Mizuno D, Higuchi K, Sakamoto T, Nakanishi H, et al. (2003). Three nicotianamine synthase genes isolated from maize are differentially regulated by iron nutritional status. Plant Physiol. 132: 1989-1997.

Pianelli K, Mari S, Marques L, Lebrun M, et al. (2005). Nicotianamine overaccumulation confers resistance to nickel in Arabidopsis thaliana. Transgenic Res. 14: 739-748.

Shojima S, Nishizawa NK and Mori S (1989). Establishment of a cell-free system for the biosynthesis of nicotianamine. Plant Cell Physiol. 30: 673-677. 
Suzuki K, Higuchi, K, Nakanishi H, Nishizawa NK, et al. (1999). Cloning of nicotianamine synthase genes from Arabidopsis thaliana. Soil Sci. Plant Nutr. 45: 993-1002.

Takahashi M, Terada Y, Nakai I, Nakanishi H, et al. (2003). Role of nicotianamine in the intracellular delivery of metals and plant reproductive development. Plant Cell 15: 1263-1280.

Vacchina V, Mari S, Czernic F, Marques L, et al. (2003). Speciation of nickel in a hyperaccumulating plant by high-performance liquid chromatography-inductively coupled plasma mass spectrometry and electrospray MS/MS assisted by cloning using yeast complementation. Anal. Chem. 75: 2740-2745.

Yang G, Li J, Liu W, Yu Z, et al. (2015). Molecular cloning and characterization of MxNAS2, a gene encoding nicotianamine synthase in Malus xiaojinensis, with functions in tolerance to iron stress and misshapen flower in transgenic tobacco. Sci. Hort. 183: 77-86.

Zhi Y, Deng Z, Luo M, Ding W, et al. (2015). Influence of heavy metals on seed germination and early seedling growth in Eruca sativa Mill. Am. J. Plant Sci. 6: 582-590.

Zhou ML, Qi LP, Pang JF, Zhang Q, et al. (2013). Nicotianamine synthase gene family as central components in heavy metal and phytohormone response in maize. Funct. Integr. Genomics 13: 229-239. 